\title{
Original
}

\section{An Application of the Mechanics of Fluid-Filled Poroelastic Solids -Ink Flow Mechanism in Mimeographing-}

\author{
by \\ Michio Kurashige ${ }^{\dagger}$, Hironobu Takasawa ${ }^{\dagger \dagger}$ and Tomiya Mori ${ }^{\dagger \dagger}$
}

\begin{abstract}
To understand main ink transfer mechanisms in mimeographing, the theory of poroelasticity is applied to analyze the ink flow through screens and a printing master which are modeled by a fluid-filled poroelastic single layer. The layer is assumed to be pressed by a press roller from below and to be subjected from above to the hydrodynamic lubrication pressure induced between a printing drum and an ink roller. The deformation of the layer and the accompanying ink flow are shown to be governed by four nondimensional parameters newly defined here. The numerical results show some important results ; e.g., squeezing-out of the ink from the layer by the layer compaction by the press roller plays an important role among various possible ink transfer mechanisms, especially for the case where the compression duration is small in comparison with the characteristic time of the layer.
\end{abstract}

Key Words : Proelasticity, Darcy Flow, Porous Material, Mimeograph, Printing Technology, Ink Flow, Squeezing, Printing Master, Screen

\section{Introduction}

In mimeographing, the mechanisms of ink transfer through printing drum perforations, screens, a master paper base, and a master polyester film to a sheet of printing paper have not completely been understood yet. This understanding may be useful to improve rotary mimeograph printers and innovate in their design.

As possible fundamentel causes of the ink transfer, the following may be pointed out: (1) the ink pressure rise induced by a hydrodynamic lubrication effect between the printing drum and the ink roller which rotate with different peripheral speeds, (2) squeezing-out of the ink from the screenmaster layer which is compacterd by the press roller, (3) the centrifugal force acting on the ink due to rotation of the drum, (4) suction of the ink by the printing paper due to capillary action, and so on. We infer that the second cause listed above may play an important role, but this ink squeezingout caused by the compaction of the screen-master layer has never been taken into account.

In the present paper, to understand the first two causes that may play more important roles,

Received January 9, 1995

$\dagger$ Department of Mechanical Engineering, Iwate University, Morioka 020, Japan

†† Division of R \& D, Tohoku Ricoh., Miyagi-ken, Shibata-cho 989-16, Japan 
we have analyzed the ink flow through the screen-maser layer by resorting to the mehanics of fluidsaturated poroelastic solids (Rice and Cleary ${ }^{1)}, 1976$; Cleary ${ }^{2)}$, 1977); this ink-filled layer has been regarded as a fluid-saturated poroelastic solid with incompressible constituents (Kurashige ${ }^{3)}$, 1982). It has been assumed that the layer is subjected from above to the pressure induced by hydrodynamic lubrication effect $\left(\right.$ Gohar $^{4)}$, 1988) and from below to the contact pressure of Hertzian type (Timoshenko and Goodier $\left.{ }^{5}, 1951\right)$. The analysis has started with seeking indicial admittances to a unit pressure rise at the top of the layer and to unit total normal traction at its bottom, and then we have obtained the ink out-flux from the master by the Duhamel integrals and the total ink volume transferred to the printing paper by integration of the out-flux. From this analysis, we have had some important conclusions about the ink transfer mechanisms.

\section{Schematic of Drum and Model for Analysis}

A schematic of a printing part of the mimeograph printer is shown in Fig. 1. The drum is made of stainless steel and perforated. It is wound around by two screens and a printing master composed of a Japanese paper base and a polyester film (stencil). Only when printing, an ink roller is pressed

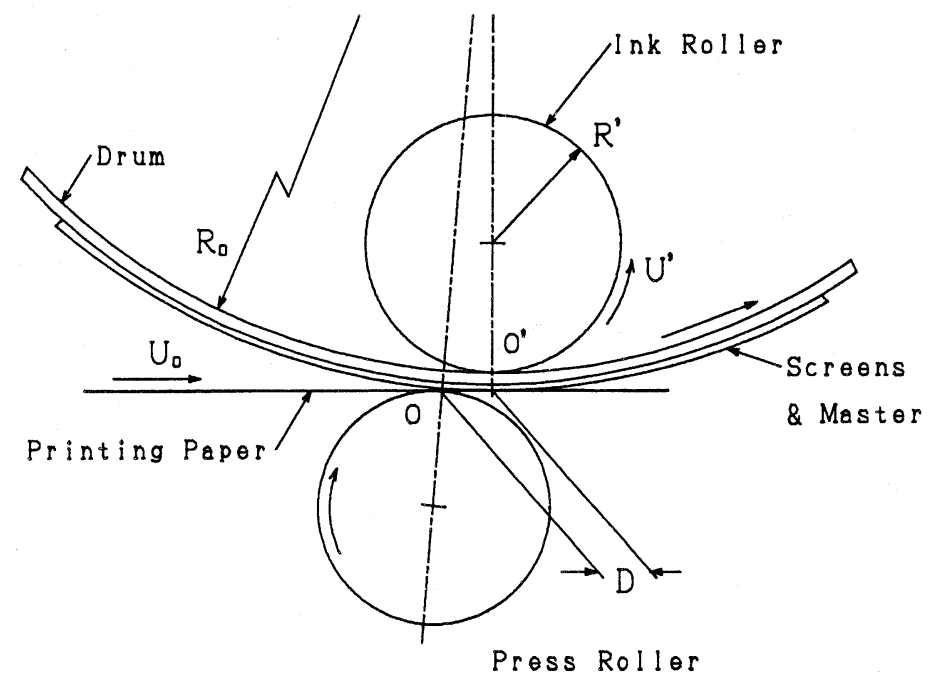

Fig. 1 Schematic of a printing part of the drum

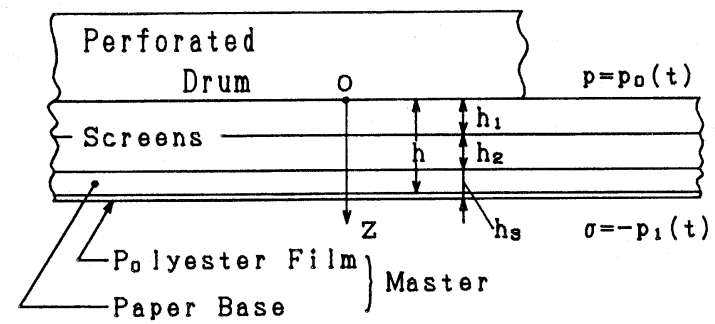

Fig. 2 Model for analysis (screens and a master regarded as a "single" fluid-saturated poroelastic layer) 
to the drum from its inside, and a press roller from the outside. A sheet of printing paper goes through between the screen-master layer and the press roller. Ink is supplied from an upper part of the ink roller, and carried by rotation of the ink roller into between the drum and the ink roller. The ink pressurized by a hydrodynamic lubrication effect flows through small perforations of the drum and then through the screens and the master, and is finally transferred to the sheet of printing paper.

The drum is so more rigid than the screens and the master that the deformation of the drum can be neglected. The radius of the drum is much larger than the thickness of the screens and the master, so that we regard the drum, the screens, and the master as plane layers (i. e., layers without curvature), when we analyze the ink flow. See Fig. 2. We regard the screens and the master's paper base as fluid-saturated poroelastic layers to take into account the effect of the compaction of the layers.

Furthemore, although the screens and the master have different elastic moduli and permeabilities, we regard a combination of these as a "single" fluid-saturated poroelastic layer by introducing an effective elastic modulus and an effective permeability.

Compliance of the layers is much larger than compressibility of layers' constituents themselves so that we can apply the incompressible model of the poroelasticity theory to the layer (Kurashige ${ }^{3)}$, 1982).

Furthermore, we consider that the top of the layer is subjected to some part of the pressure induced between the drum and the ink roller by the hydrodynamic lubrication effect, while its bottom is subject to the contact pressure of the Hertzian type. The both pressures move on the upper and lower surfaces of the layer with rotation of the drum. However, we assume that the layer experiences the spatially uniform pressure change over its whole surfaces which varies with time in shape of the pressure distributions of the hydrodynamic lubrication and the Hertzian contact. Thus, the problem for the present analysis is reduced to a time-dependent one-dimensional problem.

Before proceeding to the analysis, it should be mentioned that the layer composed of the two screens and the master shows nonlinear elasticity and that the permeability of the layer depends on the layer deformation. However, we will employ the linear theory of poroelasticity for the sake of simplicity.

\section{Basic Equations}

Since the problem under consideration is spatially one-dimensional problem, the basic equations for the irrotational displacement field $\left(\mathrm{Cleary}^{2)}, 1977\right)$ are applicable to the present analysis. For the incompressible model that we will employ below, Poisson's ratio in the undrained state and Skempton's constant are $\nu_{\mathrm{u}}=0.5$, and $\mathrm{B}=1$, respectively, in the theory of mechanics of fluid-saturated poroelastic solids (Rice and Cleary ${ }^{1)}, 1976$ ). Thus, if we introduce the z-axis downward with its origin at the top of the layer, the constitutive equations are

$$
\sigma=\mathbf{E}^{\prime} \varepsilon-\mathbf{p}, \quad \mathbf{q}=-\kappa \partial \mathbf{p} / \partial \mathbf{z}
$$

with total stress $\sigma=\sigma(\mathrm{z}, \mathrm{t})$, average strain $\varepsilon=\varepsilon(\mathrm{z}, \mathrm{t})$, pore fluid pressure $\mathbf{p}=\mathbf{p}(\mathrm{z}, \mathrm{t})$, and volume flux $q=q(z, t)$, where $\kappa$ is the permeability. $E^{\prime}$ is the elastic modulus for the uniaxial deformation; if the material is isotropic, $E^{\prime}=E(1-\nu) /(1+\nu)(1-2 \nu)$ with $\mathbf{E}$ and $\nu$ being Young's modulus and Poisson's ratio. For the case of no body force, the equilibrium equation is

$$
\partial \sigma / \partial \mathbf{z}=\mathbf{0}
$$


and the pore fluid diffusion equation is

$$
\partial \mathbf{p} / \partial \mathbf{t}=\kappa \mathbf{E}^{\prime} \partial^{2} \mathbf{p} / \partial \mathbf{z}^{2}-\mathbf{E}^{\prime} \mathbf{d g} / \mathbf{d t}
$$

where $g(t)$ is the function of time only given by

$$
\mathrm{g}(\mathrm{t})=\varepsilon-\mathbf{p} / \mathbf{E}^{\prime} \text {. }
$$

The relation between the strain and the displacement $u=u(z, t)$ is

$$
\varepsilon=\partial \mathbf{u} / \partial \mathbf{z} \text {. }
$$

The above five equations are a complete system of differetial equations together with the appro priate initial and boundary conditions. In the present analysis, to regard the layer composed of the screens and the master as a "single" fluid-saturated porous elastic layer, we will introduce the effect ive elastic modulus and permeability defined by

$$
\begin{aligned}
\kappa & =\mathbf{1} /\left\{\left(\mathbf{h}_{1} / \mathbf{h}\right) / \kappa_{1}+\left(\mathbf{h}_{2} / \mathbf{h}\right) / \kappa_{2}+\left(\mathbf{h}_{3} / \mathbf{h}\right) / \kappa_{3}\right\}, \\
\mathbf{E}^{\prime} & =\mathbf{1} /\left\{\left(\mathbf{h}_{1} / \mathbf{h}\right) / \mathbf{E}^{\prime}{ }_{1}+\left(\mathbf{h}_{2} / \mathbf{h}\right) / \mathbf{E}_{2}{ }_{2}+\left(\mathbf{h}_{3} / \mathbf{h}\right) / \mathbf{E}^{\prime}{ }_{3}\right\},
\end{aligned}
$$

where $h_{i}, \kappa_{i}$, and $E_{i}^{\prime}$ denote thickness, permeability, and an elsastic modulus for the screens $(i=1,2)$ and the master $(i=3)$, and $h=h_{1}+h_{2}+h_{3}$. See Fig. 2. The ink out-flux from the layer bottom and the transferred ink volume to the paper evaluated by use of these effective modulus and permeatility have agreed very well to those estimated bor three different layers having the real different moduli and permeabilities obtained experimentally. From this reason, we will use these effective modulus and permeability in what follows.

\section{Responses to Step Loads}

To obtain the the responses of the layer to (a) the time-dependent pressure induced by the hydrodynamic lubrication effect on its upper surface and (b) the pressure varying with time in form of the contact pressure distribution, we shall start with obtaining the indicial responses to unit step inputs.

Once these indicial responses are known, the real responses are easily obtained by the Duhamel integrals.

The governing equations are given by Eqs. (1) through (5), and the associated initial conditions are those that all dependents are zero at time $\mathrm{t}=\mathbf{0}$ for the both cases. For the case (a), the boundary conditions are

$$
\begin{array}{cl}
\mathbf{u}=\mathbf{0}, \quad \mathbf{p}=\mathbf{H}(\mathrm{t}) & \text { at } \mathrm{z}=\mathbf{0}, \\
\sigma=\mathbf{0}, \quad \mathbf{p}=\mathbf{0} & \text { at } \mathrm{z}=\mathrm{h},
\end{array}
$$

where $H(t)$ denotes the Heaviside unit step function. The first condition indicates that the layer does not part from the drum because it is wound around the drum, while the second equation in (8) indicates that the pore fluid pressure at the layer top is equal to the applied pressure. For the case (b), they are

$$
\begin{array}{ll}
\mathbf{u}=\mathbf{0}, \mathbf{p}=\mathbf{0} & \text { at } \mathrm{z}=\mathbf{0}, \\
\sigma=-\mathrm{H}(\mathrm{t}), \mathbf{p}=\mathbf{0} & \text { at } \mathrm{z}=\mathrm{h} .
\end{array}
$$

The two conditions in (11) allow for contact and free drainage at the layer bottom. The free drainage comes from the assumptions the polyester film does not prevent the ink from flowing out (because we consider the case in which the master is fully cut (full cut stencil) and that the printing paper neither foster nor hinders the ink transfer.

Solving the ordinary differential equations (with respect to $\mathrm{z}$ ) resulting from the Laplace transforms of the governing equations (1) through (5), and applying to the solutions the transformed 
boundary conditions given by Eqs. (8) and (9) or Eqs. (10) and (11) to determine integral constans, one can obtain the volume out-flux from the lower surface $(z=h)$ of the layer (that is, the indicial admittances); the out-flux for the case (a) is

$$
\mathbf{q}_{0}(\tau) /(\kappa / \mathbf{h})=2 \sum_{\mathrm{n}=0}^{\infty} \varepsilon_{\mathrm{n}}(-\mathbf{1})^{\mathrm{n}} \mathbf{e}^{-(\mathrm{n} \pi)^{2} \tau},
$$

and for the case (b), it is given by

$$
\mathbf{q}_{1}(\tau) /(\kappa / \mathbf{h})=2 \sum_{\mathrm{n}=1,3, * *}^{\infty} \mathbf{e}^{-(\mathrm{n} \pi)^{2} \tau},
$$

with symbol $\varepsilon_{\mathrm{n}}=1 / 2$ for $\mathrm{n}=0$ and 1 for $\mathrm{n} \geq 1$ and the nondimensional time being

$$
\tau=\mathbf{t} / \mathbf{t}_{\mathrm{c}}
$$

where $t_{c}$ is the characteristic time of the fluid-saturated poroelastic layer defined by

$$
\mathbf{t}_{\mathrm{c}}=\mathbf{h}^{2} /\left(\kappa \mathbf{E}^{\prime}\right) \text {. }
$$

\section{Contact Pressure}

The contact pressure between the press roller and the drum, although the screens, the master and the printing paper are inserted between them, may be estimated by the theory of Hertzian contact (Timoshenko and Goodier ${ }^{5)}$, 1951). When the maximum contact pressure and the width of contact are denoted by $\mathrm{P}_{\mathrm{m}}$ and $\mathrm{x}_{1}$, the contact pressure distribution is

$$
\mathbf{p}_{1}(\mathbf{x})=4 \mathbf{P}_{\mathrm{m}} \mathbf{x}\left(\mathbf{x}_{1}-\mathbf{x}\right) / \mathbf{x}_{1}{ }^{2},
$$

where $\mathrm{x}$ means the coordinate measured from the edge of contact area into the contact region. The maximum pressure $P_{m}$ can be known by the Hertzian theory, either if the total load exerted on the drum by the press roller, and the contact width (and the length of the roller) are experimentally measured, or if the total load, the radii of the drum and the press roller, and their elastic properties are given. As the drum and the roller rotate, any point on the bottom surface of the layer experiences the contact pressure varination given by, as can be seen from Eq. (16),

$$
\mathbf{p}_{1}(\tau) / \mathbf{P}_{\mathrm{m}}=4 \tau\left(\tau_{1}-\tau\right) / \tau_{1}{ }^{2},
$$

where $\tau_{1}$ is the nondimensionalized time duration of contact defined by

$$
\tau_{1}=\mathbf{t}_{1} / \mathbf{t}_{\mathbf{c}}=\left(\mathbf{x}_{1} / \mathbf{U}_{0}\right) / \mathbf{t}_{\mathrm{c}}
$$

with $\mathrm{U}_{0}$ being the common peripheral speed of the drum and the roller. See Figs. 1 and 3.

\section{Pressure induced between Ink Roller and Drum}

Distribution of the pressure induced between the drum and the ink roller by the hydrodynamic lubrication effect can be estimated by solving the one-dimensional Reynolds equation. We shall borrow the results from a text book on elastohydrodynamics (Gohar ${ }^{4}$, 1988). The drum of radius $R_{0}$ and the ink roller of radius $R^{\prime}$ are in line contact and in a rolling-sliding motion with the respective speeds $U_{0}$ and $U^{\prime}$. To estimate the pressure distribution, the drum and the roller are replaced by an equivalent cylinder of the reduced radius $R$ and peripheral speed $U$ defined by

$$
\mathrm{R}=1 /\left(1 / \mathrm{R}^{\prime}-1 / \mathrm{R}_{0}\right), \quad \mathrm{U}=\mathrm{U}^{\prime}+\mathrm{U}_{0},
$$

For this equivalent cylinder, if one uses the SS condition (also known as the Reynolds exit condition) as a more realistic exit condition, the distribution is given by

$$
\overline{\mathbf{p}}(\overline{\mathbf{x}})=-\frac{\left(1+\mathbf{c}_{0}^{2}\right) \overline{\mathbf{x}}}{4\left(1+\overline{\mathbf{x}}^{2}\right)^{2}}+\frac{1-3 \mathrm{c}_{0}^{2}}{8}\left[\frac{\overline{\mathbf{x}}}{1+\overline{\mathbf{x}}^{2}}+\tan ^{-1}(\overline{\mathbf{x}})+\frac{\pi}{2}\right],
$$


with the non-dimensional coordinate and pressure being defined by

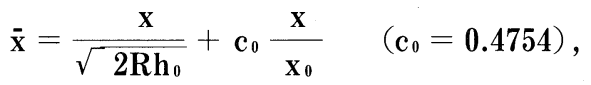

$$
\begin{aligned}
& \overline{\mathbf{p}}=\frac{\mathrm{h}_{0}{ }^{2} \mathrm{p}}{6 \sqrt{2 \mathrm{Rh}_{0} \mathrm{U} \eta_{0}}}=\mathrm{c}_{1} \frac{\mathrm{p}}{\mathrm{p}_{\mathrm{m}}} \quad\left(\mathrm{c}_{1}=0.1267\right),
\end{aligned}
$$

where $\mathrm{x}_{0}$ is the forward deviation of the pressure peak point from the contact line (see Fig. 3) and $\mathrm{p}_{\mathrm{m}}$ is the maximum ink pressure, while $\eta_{\mathrm{o}}$ being the viscosity of the ink. If the total load exerted

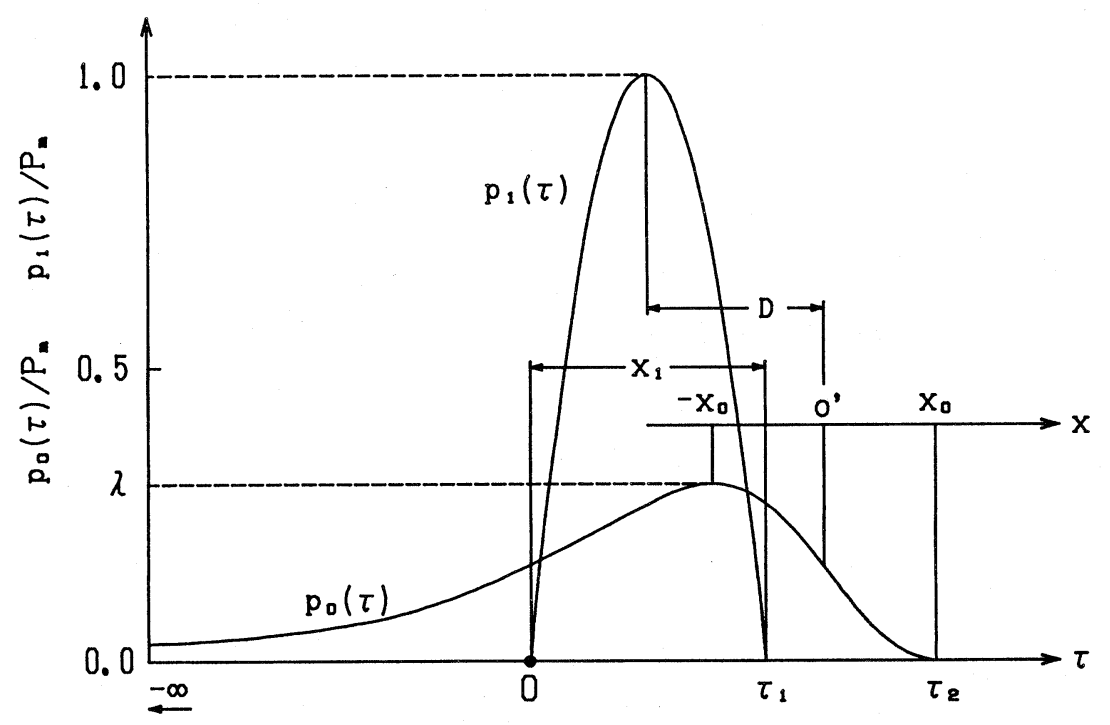

Fig. 3 Pressures exerting on the upper and lower surfaces of the layer

on the drum by the ink roller and the length of the roller are denoted by $\mathrm{W}^{\prime}$ and $\mathrm{L}^{\prime}$, the minimum ink film thickness denoted by $h_{0}$ is given by

$$
\mathrm{h}_{0}=\mathrm{c}_{2} \frac{\mathrm{U} \eta_{0} \mathrm{RL}^{\prime}}{\mathrm{W}^{\prime}} \quad\left(\mathrm{c}_{2}=2.45\right) \text {. }
$$

If the total load is known experimentally, the minimum film thickness can be estimated by the above equation, and vice visa.

Estimation of the pressure distribution by Eq. (20) is exact within the framework of the elastohydrodynamic lubrication theory, provided that the drum is not perforated. It may be important or crucial how to cope with the influence of the perfoations on the pressure. We assume that the pressure which acts on the upper surface of the layer is the same in distribution as and $\alpha$ times the pressure induced between the drum and the ink roller $(\alpha<1)$. Thus, the parameter $\alpha$ reflects the pressure drop caused by the perforations, the pressure drop during the ink flowing through the perforaions, and the effect of perforation area proportion to the whole area of effective drum surface as well . The ratio of the maximum pressure at the upper layer surface to the maximum contact pressure at the lower surface, if denoted by $\lambda$, is expressed as

$$
\lambda=\frac{\alpha \mathbf{p}_{\mathrm{m}}}{\mathbf{P}_{\mathrm{m}}}=\mathrm{c}_{1} \alpha \frac{6 \sqrt{2 \mathrm{R} \mathbf{h}_{0} \mathrm{U} \eta_{0}}}{\mathbf{P}_{\mathrm{m}} \mathbf{h}_{0}{ }^{2}} .
$$


The contact line between the drum and the press roller is deviated from that between the drum and the ink roller ; this deviation is called an "off-set" of the press roller and denoted by D. See Fig. 1. Taking into account this deviation, one finds the relation of the non-dimensional coordinate $\bar{x}$ to the non-dimensional time $\tau$ as follows (see Fig.3) :

$$
\overline{\mathbf{x}}=\mathbf{c}_{0}\left(\tau-\tau_{0} \Delta-\tau_{1}\right) / \tau_{0},
$$

where

$$
\begin{aligned}
& \Delta=\frac{\mathrm{D}}{\mathrm{x}_{0}}=\frac{\mathrm{D}}{\mathrm{c}_{0} \sqrt{2 \mathrm{Rh_{0 }}}}, \\
& \tau_{0}=\frac{\mathbf{x}_{0} / \mathrm{U}_{0}}{\mathrm{t}_{0}}=\frac{\mathrm{c}_{0} \sqrt{2 R \mathbf{h}_{0} / \mathrm{U}_{0}}}{\mathbf{h}^{2} /\left(\kappa / \mathbf{E}^{\prime}\right)} .
\end{aligned}
$$

With Eq. (26), $\Delta=1$ implies that the peaks of the pressures applied on the both surfaces coincide each other in position.

Although there have appeared many material parameters and dimensions of the printer in the above analysis, only the four non-dimensional parameters given by Eps. (18), (24), (26), and (27) govern the problem under consideration. That is, if these four parameters are given, one can estimate the out-flux of the ink from the layer, the ink volume transferred to the printing paper, and others in terms of the respective non-dimensional quantities.

Finally, taking into account Eqs. (24) and (25), one obtains the pressure variation on the upper surface of the layer as follows :

$$
\mathbf{P}_{0}(\tau) / \mathbf{P}_{\mathbf{m}}=\left(\lambda / \mathbf{c}_{1}\right) \overline{\mathbf{p}},
$$

where $\overline{\mathrm{p}}$ is given by Eq. $(20)$.

\section{Ink Out-Flux}

We now know the pressure variations, $\mathbf{p}_{0}(\tau)$ and $\mathbf{p}_{1}(\tau)$, on the upper and lower surfaces and the corresponding inditial responses, $q_{0}(\tau)$ and $q_{1}(\tau)$, to the unit inputs ; thus, we can calculate the ink out-flux from the lower surface by the Duhamel integrals. The out-flux is given by

$$
\frac{\mathbf{Q}(\tau)}{\mathbf{P}_{\mathrm{m}} \kappa / \mathbf{h}}=\int_{-\infty}^{\tau \mathbf{q}_{0}}(\tau-\gamma) \frac{\mathbf{d}}{\mathbf{d} \gamma} \mathbf{p}_{0}(\gamma) \mathbf{d} \gamma+\int_{0}^{\tau(1)} \mathbf{q}_{1}(\tau-\gamma) \frac{\mathbf{d}}{\mathbf{d} \gamma} \mathbf{p}_{1}(\gamma) \mathbf{d} \gamma,
$$

where, if $\tau_{1}<\tau_{2} \equiv \tau_{0}(1+\Delta)+\tau_{1} / 2$,

$$
\begin{aligned}
& \tau^{(0)}=\tau, \quad \tau^{(1)}=\mathbf{0} \quad \text { for }-\infty<\tau \leq \mathbf{0}, \\
& \tau^{(0)}=\tau, \quad \tau^{(1)}=\tau \quad \text { for } \quad 0<\tau \leq \tau_{1} \text {, } \\
& \tau^{(0)}=\tau, \quad \tau^{(1)}=\tau_{1} \text { for } \quad \tau_{1}<\tau \leq \tau_{2} \text {, } \\
& \tau^{(0)}=\tau_{2}, \quad \tau^{(1)}=\tau_{1} \text { for } \tau_{2}<\tau \leq \infty \text {, }
\end{aligned}
$$

and if $\tau_{1}>\tau_{2}$,

$$
\begin{aligned}
& \tau^{(0)}=\tau, \quad \tau^{(1)}=\mathbf{0} \quad \text { for }-\infty<\tau \leq \mathbf{0} \text {, } \\
& \tau^{(0)}=\tau, \quad \tau^{(1)}=\tau \quad \text { for } \quad 0<\tau \leq \tau_{2} \text {, } \\
& \tau^{(0)}=\tau_{2}, \quad \tau^{(1)}=\tau \text { for } \quad \tau_{2}<\tau \leq \tau_{1} \text {, } \\
& \tau^{(0)}=\tau_{2}, \quad \tau^{(1)}=\tau_{1} \text { for } \tau_{1}<\tau \leq \infty,
\end{aligned}
$$

because $\mathbf{p}_{0}(\tau)$ vanishes only for $\tau_{2}<\tau$ and $\mathbf{p}_{\mathbf{1}}(\tau)$ is zero for $\tau<0$ and for $\tau_{1}<\tau$. See Fig. 3 . 
Substitution of Eqs. (12) and (13) into Eq. (29) and integration of the resulting equation by parts yields

$$
\begin{aligned}
\frac{\mathbf{Q}(\tau)}{\mathbf{P}_{\mathrm{m}} \kappa / \mathbf{h}}=\mathbf{P}_{\mathbf{0}}\left(\tau^{(0)}\right)+2 \sum_{\mathrm{n}=1}^{\infty}(-\mathbf{1})^{\mathrm{n}} \frac{\mathbf{e}^{-(\mathrm{n} \pi)^{2} \tau}}{(\mathbf{n} \pi)^{2}}\left[\mathbf{e}^{(\mathrm{n} \tau)^{2} \tau^{(0)}} \frac{\mathbf{d}}{\mathbf{d} \gamma} \mathbf{p}_{0}\left(\tau^{(0)}\right)-\int_{-\infty}^{\mathbf{e}^{\tau(\mathrm{n} \tau) 2 \gamma}} \frac{\mathbf{d}^{2}}{\mathbf{d} \gamma^{2}} \mathbf{p}_{0}(\gamma) \mathbf{d} \gamma\right] \\
+\frac{8}{\tau_{1}^{2}} \sum_{\mathrm{n}=1,3, * *}^{\infty} \frac{\mathbf{e}^{-(\mathrm{n} \pi)^{2} \tau}}{(\mathbf{n} \pi)^{2}}\left[\mathbf{e}^{\left(\mathrm{n} \pi^{2} \tau^{(1)}\right.}\left(\tau_{1}-2 \tau^{(1)}\right)-\tau_{1}+\frac{2}{(\mathbf{n} \pi)^{2}}\left(\mathbf{e}^{(\mathrm{n} \pi)^{2} \tau^{(1)}}-1\right)\right],
\end{aligned}
$$

where it is difficult to analytically evaluate the integral in the first square brackets of the right side, leaving it to numerical integration.

\section{Transferred Ink Volume}

Next, we obtain the ink volume transferred from the master to the printing paper by integrating the ink out-flux over the time duration of press roller pressing onto the drum. The transferred volume estimated by such a way may not be substantially different from the exact or real transferred ink volume, although we neglect the capillary effect of the printing paper, the mechanism of ink separation between the master and the printing paper, and so on.

The transferred ink volume $\mathrm{V}$ is evaluated by the following integral :

$$
\frac{\mathbf{V}}{\mathbf{P}_{\mathrm{m}} \mathbf{h} / \mathbf{E}^{\prime}}=\int_{0}^{\tau_{1}} \frac{\mathbf{Q}(\tau)}{\mathbf{P}_{\mathrm{m}} \kappa / \mathbf{h}} \mathbf{d} \tau,
$$

the integration of which also shall be numerically carried out.

\section{Numerical Calculations and Results}

We have carried out some numerical calculations using Eq. (32) for the ink out-flux from the master and Eq. (33) for the transferred ink volume. As numerical volues of the four non-dimensional characteristic parameters, we have used $\tau_{0}=0.5, \tau_{1}=1, \Delta=1$, and $\lambda=0.2$ for most cases except when they are independent variables or when examining their effect. For a commercially available rotary printer and for a specific running condition, $\tau_{0}=0.61$ and $\tau_{1}=1.12$; the non-dimensional off-set $\Delta$ is a little lager than untiy. As mentioned above, $\Delta=1$ means that the peaks of the applied pressures at the upper and lower surfaces coincide in time (or in position). The ratio, $\lambda$, of the maximum pressure at the upper surfuce to that at the lower surface is most ambiguous among the

four parameters, because we cannot know the exact value of $\alpha$ appearing in Eq. (24). However, we believe that $\lambda=0.2$ is a reasonable value, judging from our experiences.

Fig. 4 shows the time history of the ink out-flux from the master for the three time durations of compression of the layer by the press roller. Here, it should be noted that the non-dimensional time scale on the abscissa is not $\tau$ but $\tau / \tau_{1}$. The time duration of compression by the press roller begins at $\tau / \tau_{1}=0$ and ends at $\tau / \tau_{1}=1$. Before the press roller begins to compress the eayer, the ink starts to flow out from the master, although its rate is very small. Immediately after the compression (contact) begins, the ink out-flux increases rapidlly and reaches its peak. The peak is higher, the shorter the time duration of compression (i. e., the higher the peripheral speed of drum) is. At the last period of the compression duration, the ink is sucked back, as is caused by the elastic recovery of the layer. This result corresponds to the observed fact that the surface of the master is "dry" after printing. 


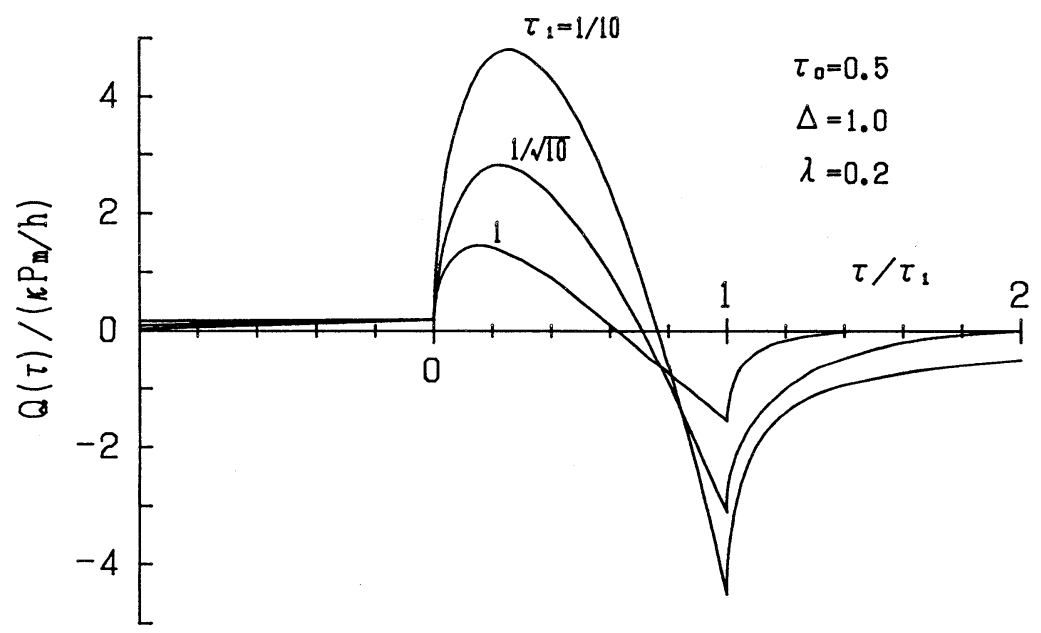

Fig. 4 Time history of the ink out-flux from the master for the three contact time durations

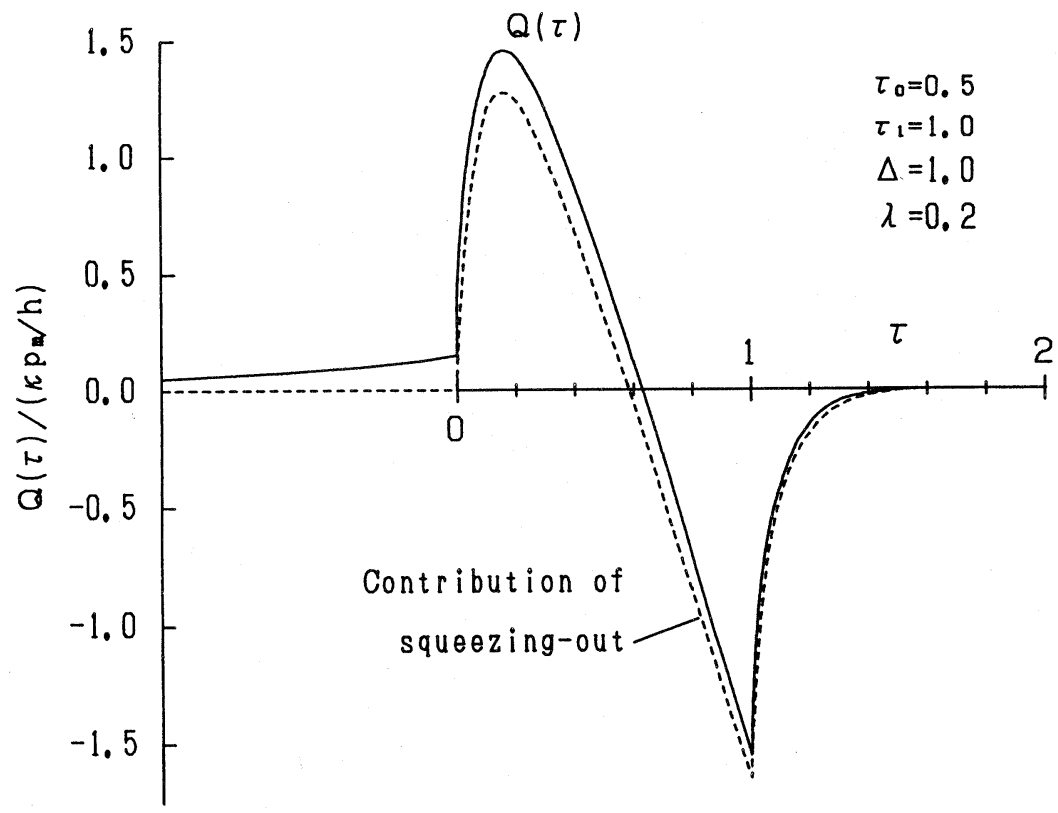

Fig. 5 Time history of the ink out-flux and contribution of the squeezing-out of ink to the flux

It is shown in Fig. 5 how much the squeezing-out of the ink by the compaction of the ink-saturated poroelastic layer pressed by the press roller contributes to the ink out-flux ; the solid line represents the total out-flux and the broken curve is for the squeezing-out contribution. For the maximum pressure ratio $\lambda=0.2$, the contribution of the squeezing-out is predominant over that of the hydrodynamic lubrication pressure to the total out-flux.

Fig. 6 depicts the ink volume transferred from the master to the printing paper (or total volume 


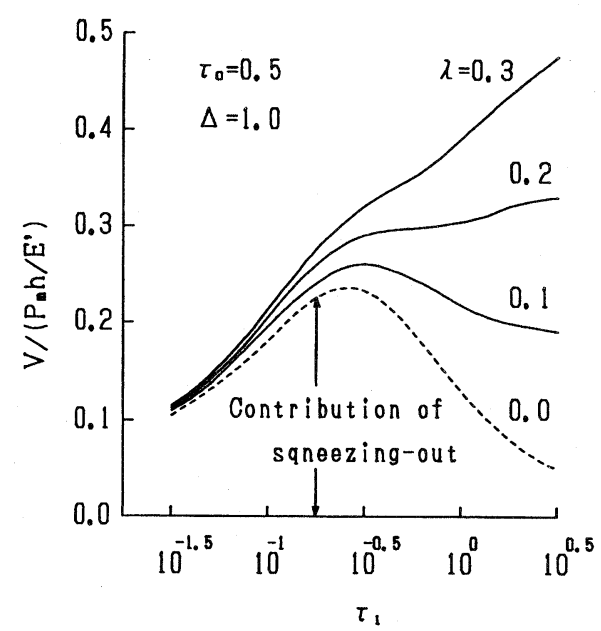

Fig. 6 Ink volume transferred from the master to the paper versus the contact time duration

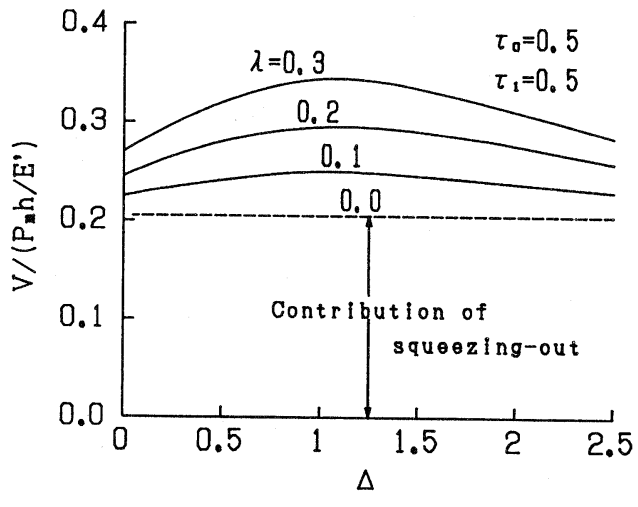

Fig. 7 Ink volume transferred from the master to the paper versus the off-set of the press roller

that flows out from the master) versus the time duration of compression of the layer for various maximum pressure ratios. For the smaller ratio, the transferred ink volume has its peak between $\tau_{1}=0.1$ and $\tau_{1}=1$. For the larger ratio, the volume has no peak but increases with the compression time duration. With a real mimeograph printer, this monotonic increase in transferred ink volume with the compression duration is expertly untilized to adjust the printing darkness.

The broken curve drawn in this figure shows again the contribution of the sqeezing-out of the ink. For the smaller time duration, this contribution prevails against that of the pressurization at the upper surface, while the latter contribution increases and becomes predominant with the increasing time duration. It may be said that, if a high speed machine is required, the effect of the squeezingout of the ink by the compaction of the layer should be expertly used.

In Fig. 7, the transferred ink volume is shown against the off-set of the press roller for various maximum pressure ratios. All curves have their peaks, although not steep, around $\Delta=1$, which implies that the peaks of the pressures at the upper and lower surfaces coincide in position. Here also shown is the contribution of the sqeezing-out effect by the broken line. For all of the pressure ratios, this contribution is more than half of the total transferred ink volume.

The time history of the out-flux is shown in Fig. 8 for $\Delta=0,1$, and 2 . Only one thing intended to show by this figure is that, for $\Delta=2$ and more, there appears the flowing-out of the ink from the master even after the press roller parts from the master. This means that a too large off-set makes the master surface "wet" after printing, probably resulting in poor printings.

\section{Conclusions}

Regarding screens and a master as a "single" ink-filled poroelastic layer, we have analyzed the mechanical behaviors of the layer and reached the following conclusions : 


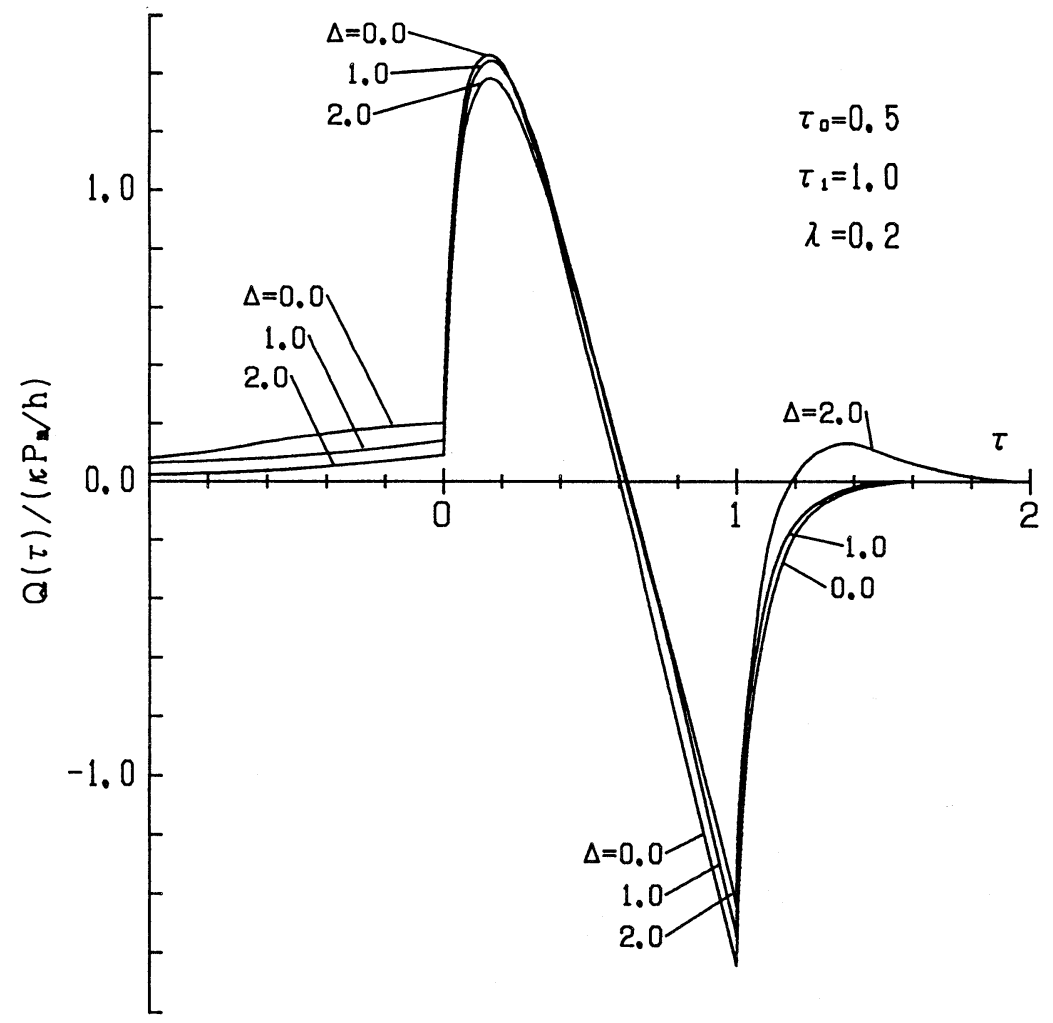

Fig. 8 Time history of the ink out-flux for the three off-set values

(1) The mechanical behaviors of the screen-master layer are governed by the four non-dimensional parameters defined by Eqs. (18), (24), (26), and (27).

(2) The squeezing-out of the ink due to the compaction of the ink-filled poroelastic layer pressed by the press roller plays an important, or rather predominant, role as a driving force among various possible causes of ink transfer, especially for the case where the compression time duration is small in comparison with the characteristic time of the layer.

(3) The ink is sucked back into the master when the contact pressure by the press roller declines, as is caused by the elastic recovery of the compacted poroelastic layer.

(4) The ink volume transferred from the master to the printing paper takes its maximum values, when the time duration of compression by the press roller is a little smaller than the characteristc time, defined by Eq. (15), of the fluid-saturated poroelastic layer, and when the off-set is approximately equal to the forward shift of the peak of the pressure induced between the drum and the ink roller by the hydrodynamic effect.

\section{Acknowledgment}

The authors wish to thank Mr. Y. Goto, Iwate University, for helping them prepare the maunscript of the paper. 


\section{References}

1) Rice, J. R. and Cleary, M.P., 1976, "Some Basic Diffusion Solutions for Fluid-Saturated Elastic Porous Media with Compressible Constituents," Reviews of Geophysics and Space Physics, Vol. 14, pp. 227-241.

2) Cleary, M. P., 1977, "Fundamental Solutions for a Fluid-Saturated Porous Solid", International Journal of Solids and Structures, Vol. 13, pp. 785-806.

3) Kurashige, M., 1982, "Transient Response of a Fluid-Saturated Poroelastic Layer Subjected to a Sudden Fluid Pressure Rise," ASME Journal of Applied Mechanics, Vol. 49, pp. 492-496.

4) Gohar, R., 1988, Elastohydrodynamics, John Wiley \& Sons, New York, pp. 79-84.

5) Timoshenko, S. P. and Goodier, J. N., 1951, Theory of Elasticity, McGraw-Hill, New York, pp. 370-382. 